\title{
Preparation of samples: Why use Ga, Xe or photons?
}

\author{
Joseph Michael
}

\section{Sandia National Laboratory, United States}

Focused ion beam (FIB) technology continues to rapidly advance and change over short periods of time. FIB tools are available with a large variety of sources and ion species. [1] The addition of femtosecond lasers to FIB tools is providing the ability to access and prepare much larger areas than were previously possible with ions alone. This talk will discuss the applications of Ga liquid metal ion source (LMIS) and Xe plasma sourced FIB tools (PFIB) as well as the general applicability of laser ablation to materials characterization.

Ga LMIS sourced FIB tools have been undergoing continuous development for the past 30 years and their applications and limitations have been established. [1] Current Ga LMIS sourced tools have been fully developed for cross section preparation for SEM imaging as well as for production of samples for TEM or STEM imaging. Current ion column developments have allowed for very small well focused Ga ion beams to be formed which have allowed site-specific and very precise sample preparation of cross sections and lamellas. The two main disadvantages of the Ga LMIS are the limited beam current, about 100nA maximum, available in a focused beam and the implantation of Ga into the sample. The probe current is limited by the physical limitations of the ion optics and the source. The low beam current determines the ultimate size of samples that can be milled in reasonable time scales. The other issue with Ga LMIS sourced FIB is the implantation of Ga into the sample. Ga can be reactive and has been shown to cause issues with a variety of materials including low melting point metals like In or segregation of $\mathrm{Ga}$ in $\mathrm{Al}$ samples. [2] [3]

Xe plasma FIB sources have become more common over the past few years. [4] [5] [6] Xe plasma FIB sourced tools can generate very high beam currents (1000's of nA) while still maintaining a focused probe. Due to the larger accessible beam currents, PFIB tools can mill much larger areas of samples in much shorter times eliminating one of the drawbacks of Ga LMIS FIB. The physics of focusing of a Xe PFIB beam does not allow the probe to be as sharply focused as a Ga beam due to the differences in the ion sources. The lower current density in the PFIB probes can result in more obvious curtaining of FIB milled surfaces than seen in Ga LMIS. The propensity to form curtains on PFIB milled surfaces can be mitigated with shadow masking, rocking the ion beam angle with respect to the surface and other milling strategies. Xe does not have the reactivity issues normally found with Ga milling. [7]

The newest addition to the FIB suite of capabilities is laser machining. [8] [9] [10] Early implementation of laser machining with a FIB used nanosecond lasers that resulted in thick heat affected zones in the sample of many micrometers. More recently, femtosecond lasers have been utilized that greatly reduce the HAZ . [11] Figure 1a shows an example of laser drilled holes in an electroformed $\mathrm{Cu}$ sample. A cross section was prepared across the two holes and EBSD was used to assess the amount of HAZ present. The thickness of the HAZ is quite thin and microstructural changes are not detectable at this resolution. The femtosecond laser ablation capability allows very large volumes of material to the removed very quickly compared to either of the ion sources at the expense of beam size and has enabled new large 3D studies of materials. [10]

Each of the milling capabilities (LMIS, PFIB or laser) have both strengths and weaknesses leading to questions about which methods are best and how to best utilize each of them in a typical laboratory setting. This presentation will cover the uses for each Ga LMIS, Xe PFIB and the femtosecond laser for sample preparation and demonstrate how a modern multi-material characterization facility may need to have access to all three types of FIB processing.

This paper describes objective technical results and analysis. Any subjective views or opinions that might be expressed in the paper do not necessarily represent the views of the U.S. Department of Energy or the United States Government. Sandia National Laboratories is a multimission laboratory managed and operated by National 
Technology and Engineering Solutions of Sandia, LLC., a wholly owned subsidiary of Honeywell International, Inc., for the U.S. Department of Energy's National Nuclear Security Administration under contract DE-NA0003525 .

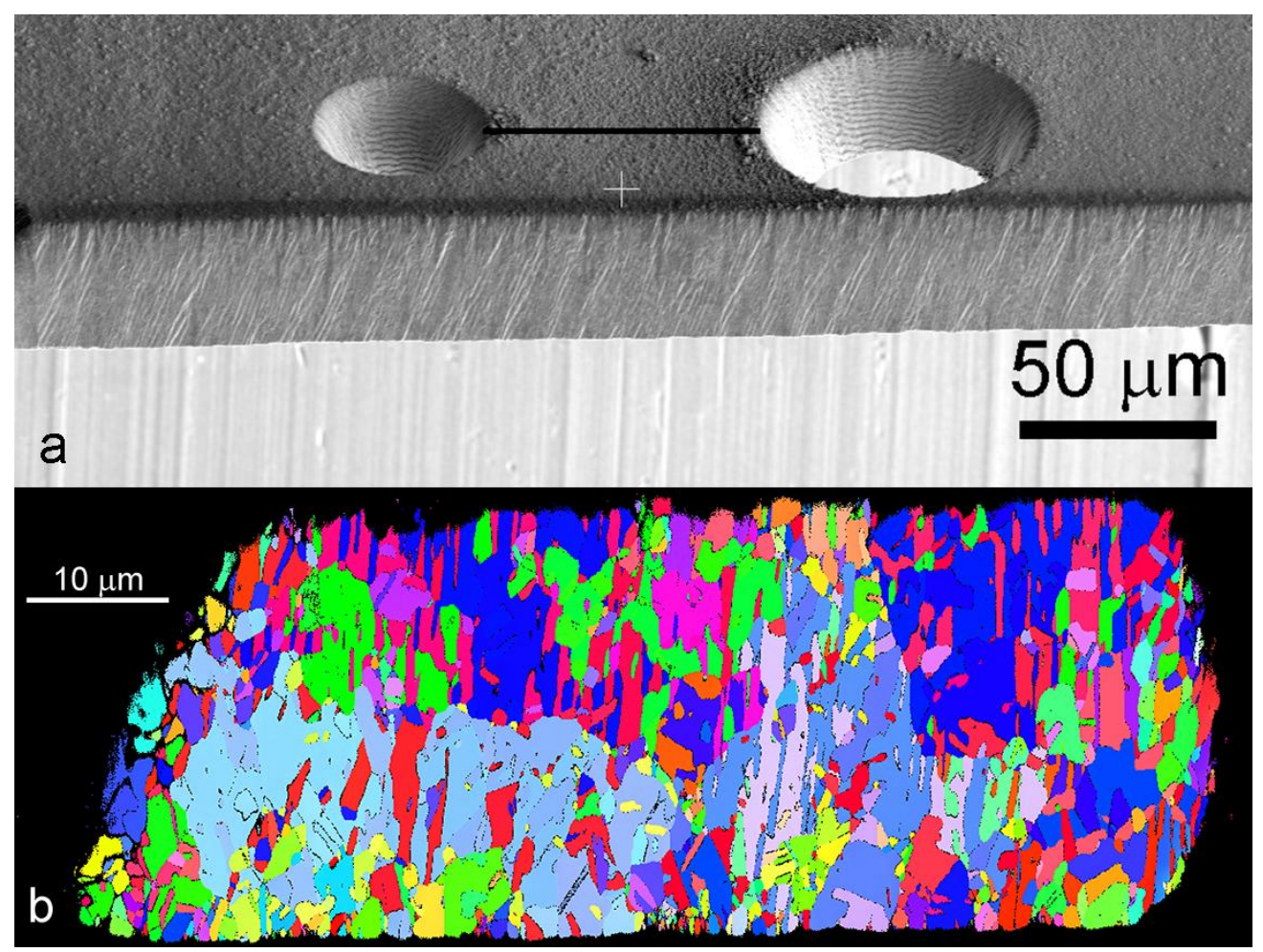

Figure 1. Laser drilled holes and cross section in a thin $\mathrm{Cu}$ electroformed sheet. a) Two $515 \mathrm{~nm}$ femtosecond laser drilled holes. b) EBSD IPF map with respect to the normal direction of the prepared cross section.

\section{References}

1. $\quad$ Bassim, N., K. Scott, and L.A. Giannuzzi, Mrs Bulletin, 2014. 39(4): p. 317-325.

2. $\quad$ Michael, J.R., Microscopy and Microanalysis, 2011. 17(3): p. 386.

3. Giannuzzi, L. and J. Michael,. Microscopy and Microanalysis, 2020. 26(S2): p. 792-793.

4. Smith, N., et al.,. Journal of Vacuum Science \& Technology B: Microelectronics and Nanometer Structures Processing, Measurement, and Phenomena, 2006. 24(6): p. 2902-2906.

5. $\quad$ Burnett, T., et al., Ultramicroscopy, 2016. 161: p. 119-129.

6. $\quad$ Burnett, T., et al., Microscopy Today, 2016. 24(3): p. 32-39.

7. Zhong, X., et al., Journal of Microscopy, 2020.

8. Randolph, S.J., et al., Journal of Vacuum Science \& Technology B, Nanotechnology and Microelectronics: Materials, Processing, Measurement, and Phenomena, 2018. 36(6): p. 06JB01.

9. Tordoff, B., et al., Applied Microscopy, 2020. 50(1): p. 1-11.

10. Echlin, M.P., et al., Current Opinion in Solid State and Materials Science, 2020. 24(2): p. 100817.

11. Valette, S., et al., Applied Surface Science, 2005. 239(3-4): p. 381-386. 